\title{
Failure Analysis of the Screw Connection Rod of A Hoisting Machine
}

\author{
Pengfei Ma1, a , Shijing Wu¹, Li Zhu¹, Wenkai Xiao ${ }^{1, *}$ \\ ${ }^{1}$ Wuhan University, School of Mechanical and Power, Engineering, Wuhan, China \\ a1059436725@qq.com, *xiaowenkai@whu.edu.cn
}

Keywords: brittle fracture; Widmanstatten structure; fracture morphology

Abstract. The screw connecting rod of a hoisting machine suddenly breaks during the service at temperatures below $20^{\circ} \mathrm{C}$. In this article, the fracture surface, material, heat treatment process and mechanical properties of the connecting rod are analyzed. We find the low temperature brittle fracture of the screw is mainly caused by too large unbalanced load due to improper operation. The incorrect heat treatment process of the screw is one of the reasons for its fracture.

\section{Introduction}

The screw connecting rod of a hoisting machine suddenly breaks during the service at temperatures below $20{ }^{\circ} \mathrm{C}$. To explore the causes of fracture and prevent such incidents from happening again, the fracture morphology, material, heat treatment process and mechanical properties of the material of the connecting rod were studied.

\section{Experimental materials and methods}

In order to reveal the cause of the fracture of the connecting rod of the suspension cylinder, the following four aspects were studied.

Fracture failure analysis of connector. The connecting rod of the hoisting machine was shown in Fig 1. Macro morphology of fracture of the connecting rod was shown in Fig 2. We will make a preliminary analysis of the reasons for the fracture of the connecting rod by analyzing the feature surface.

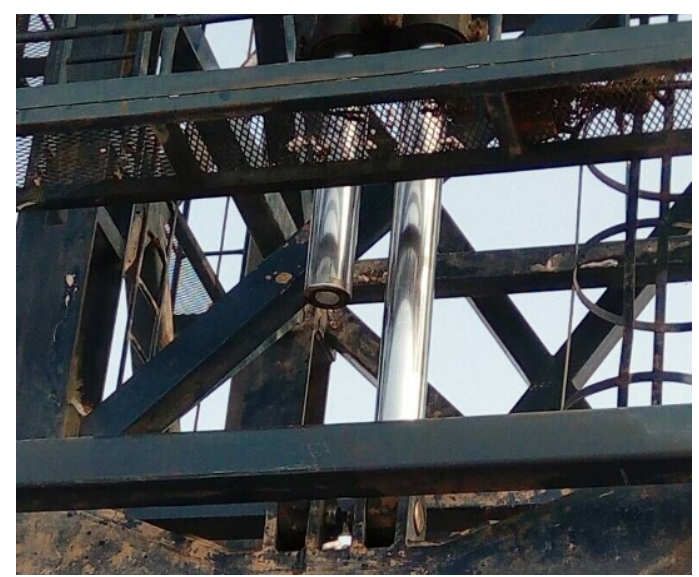

Fig 1. The connecting rod of the hoisting machine

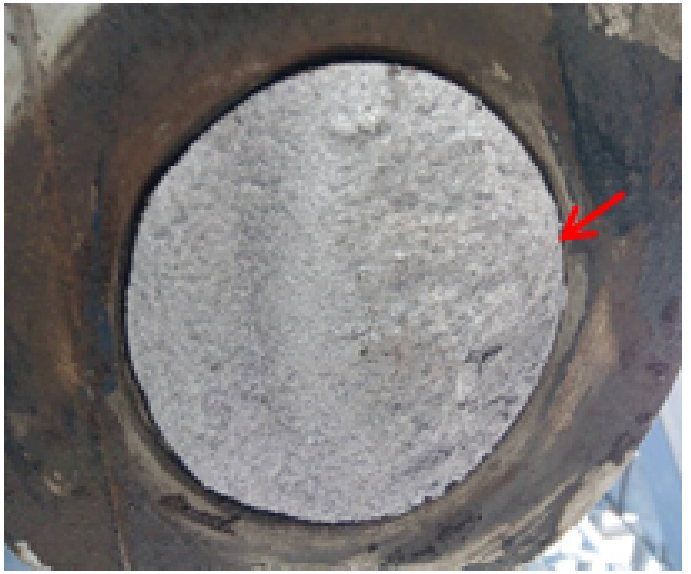

Fig 2.Fracture of the connecting rod

Material analysis.Materials are usually analyzed by chemical analysis to determine the content of carbon and sulfur accurately. The carbon and sulfur content of the samples were determined by the carbon sulfur analyzer. On the other hand, the alloy elements and their contents were determined by scanning electron microscopy .

Analysis of heat treatment process. The performance of the steel structure is closely related to the heat treatment process, so the proper heat treatment process can make the material play its proper mechanical properties.Metallographic analysis and observation were made on the prepared specimens. 
Analysis of mechanical properties of materials. The uniaxial tensile tests of two specimens were made and the stress and strain curves of the two specimens were recorded. At the end of the experiment, the shape of the fracture was analyzed.

\section{Sample test result and analysis}

Analysis of fracture surface of the connecting rod. According to Fig 2, the whole fracture was fresh and bright, and no rusty spots were found. This shows that the whole section is a new fracture. There is no old crack, which precludes the possibility of crack defects before the fracture of the connecting rod. At the same time, no shell like fatigue crack pattern was found to eliminate the possibility of fatigue fracture.

According to the radial pattern on the left side of the fracture, we determine the position of the crack source in the arrow as shown in Fig 2. Through further observation of the fracture characteristics of the sample, it is found that there are small, bright and clean cleavage cross section, and the cross section shrinkage is less than 5\%, which shows that the material is brittle cleavage fracture. Cleavage fracture is a transgranular fracture of a metallic material at a very rapid rate along a certain crystallographic plane when the normal stress of the external force reaches a certain value.The cleavage plane is generally the lowest index or the lowest surface energy. Generally, cleavage fracture is always brittle fracture, but brittle fracture is not necessarily cleavage fracture. Cleavage fracture is a typical brittle fracture. Although it is brittle fracture, there is also a crack initiation and propagation process before fracture. Cleavage fracture is a kind of low energy fracture, and the size of stress and size of grain affect cleavage fracture[1-4].

Material analysis. Scanning electron microscopy (SEM) was used to observe the metallographic spectrum of the samples. The results of SEM were shown in Fig 3. No other alloy elements are found in the sample except $\mathrm{Fe}$ and $\mathrm{C}$ elements shown in Fig 3, indicating that the joint is carbon steel. Carbon and sulfur analysis showed that carbon content (mass fraction) was $0.456 \%$ and sulfur content was $0.011 \%$. Therefore, the material used for determining the connecting piece is ordinary carbon 45 steel, and the impurity element content is nomal.

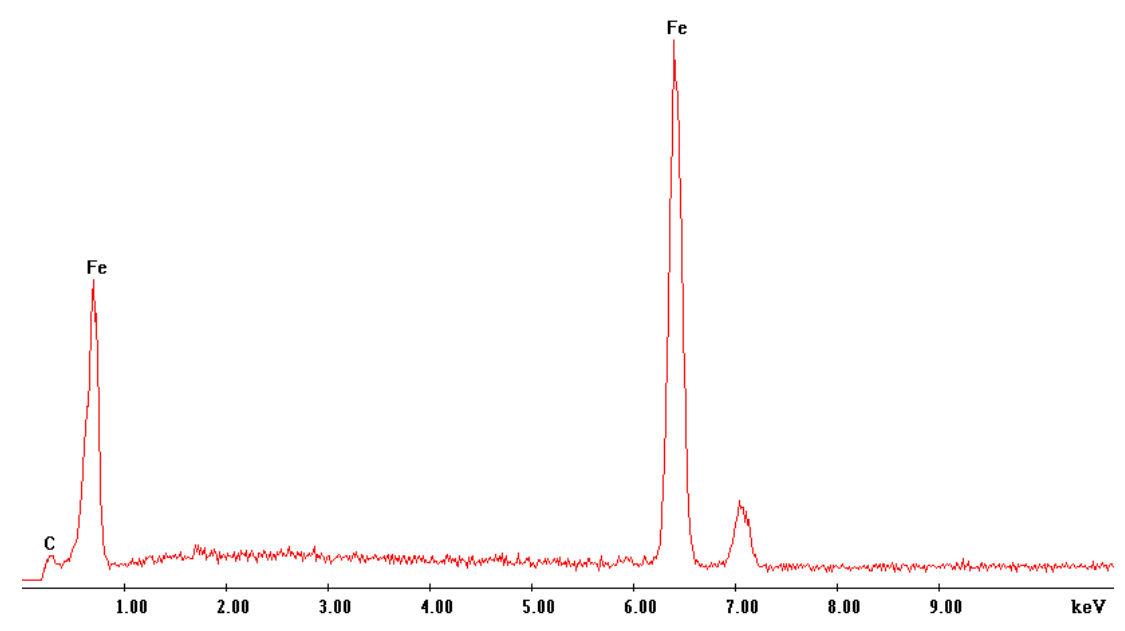

Fig 3. Sample alloy element energy spectrum

Analysis of heat treatment process for materials. Sampling is carried out on the connecting rod, followed by sanding, polishing, and etching. As shown in Fig 4, we found that the microstructure of the specimen is coarse Widmanstatten structure. The grain size in the sample is about 100-300 microns and the grain size is $0-2$. The grain of the connecting material is too large and the microstructure is poor, so the steel used for connecting parts may not be normalized and quenched and tempered. So far, the formation mechanism of Widmanstatten structure has not yet reached full consensus, but the research work has been to continue[5-6]. The Widmanstatten structure is a kind of overheating defect structure steels, which reduces the ductile brittle transition temperature of the steel, 
so fragile in the use of low temperature environment will be greatly enhanced. Experimental results show that the coarse austenite grain is more easy to produce Widmanstatten structure and fine austenite grain is not easy to produce Widmanstatten structure[7-9] .

Mechanical properties of materials. Axial tensile tests of two specimens were carried out at $10{ }^{\circ} \mathrm{C}$. The sample is stretched before and after drawing as shown in Fig 5, Fig 6. It is found that the fracture of the joint material is grey and dark at the $10{ }^{\circ} \mathrm{C}$ static tensile condition, and there is obvious necking at the fracture. It shows a plastic fracture feature. But it should be pointed out that one of the two specimens has the characteristic of brittle fracture, so it is brittle.

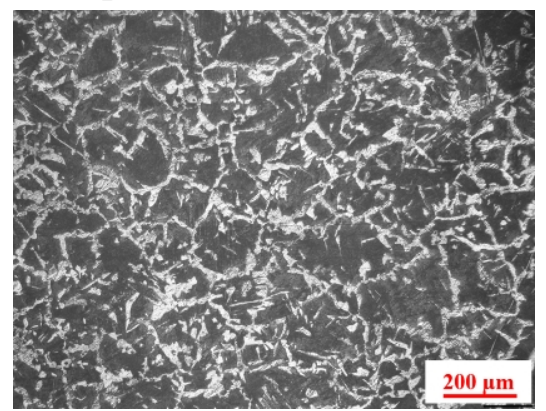

Fig 4. Metalloghic structure of sample

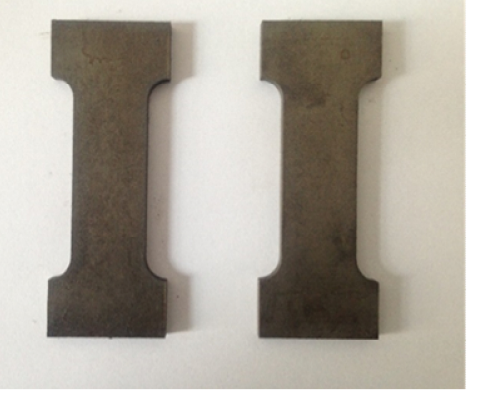

Fig 5. Sample before tension

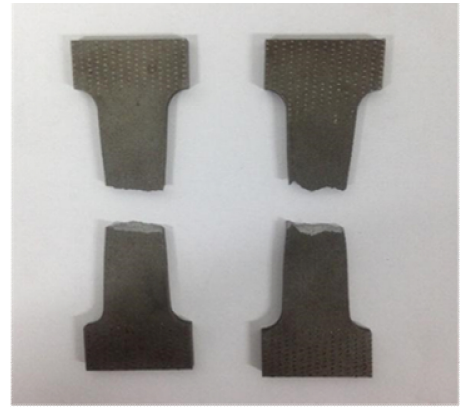

Fig 6.Sample after tension

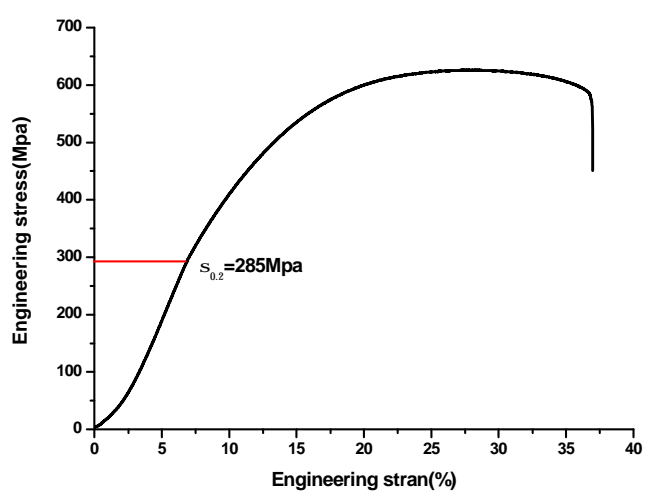

Fig 7. Tensile curves of sample A

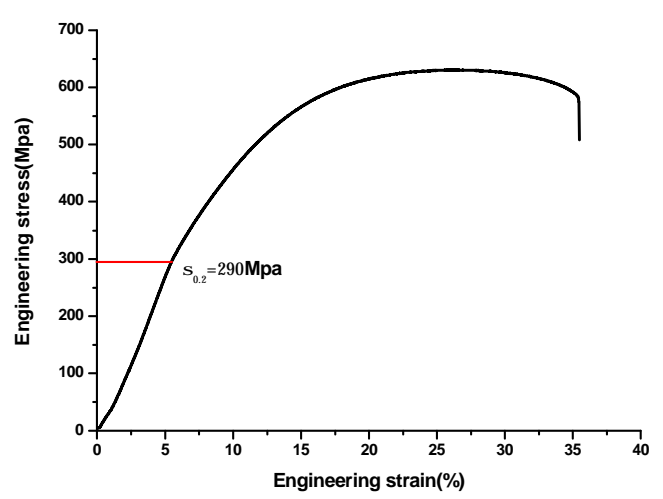

Fig 8. Tensile curves of sample B

The stress and strain curves of the two specimens have no obvious yielding platform as shown in Fig 7, Fig 8. According to the GB/T 699-2015 standard[10], the yield strength is determined by $\sigma_{0.2}$. The mechanical properties of the two specimens are shown in Tab 1.

\begin{tabular}{|c|c|c|c|c|c|}
\hline & $\sigma_{0.2}(\mathrm{Mpa})$ & $\sigma_{\mathrm{b}}(\mathrm{MPa})$ & $\varphi$ & $\delta$ & HRC \\
\hline SampleA & 285 & 620 & $33 \%$ & $36 \%$ & 15.8 \\
\hline SampleB & 290 & 625 & $35 \%$ & $36 \%$ & 16 \\
\hline
\end{tabular}

Tab 1. Mechanical properties of specimens A and B

According to the GB/T 699-2015 standard, the basic mechanical properties of 45 steel should satisfy: tensile strength is more than $600 \mathrm{MPa}$, the yield strength is above $355 \mathrm{MPa}$, the elongation is more than $16 \%$, more than $40 \%$ section shrinkage and delivery hardness more than $229 \mathrm{HB}$. It is found that the fracture connection, the screw yield strength, the cross section shrinkage and hardness are all lower by comparison. The main reason is that the heat treatment is not correct. The microstructure of the specimen is coarse Widmanstatten . which results in low number of its mechanical properties. The screw parts are not one of the important factors to produce brittle fracture at low temperature without proper heat treatment. 
But considering the temperature of the fracture of the screw is $-20^{\circ} \mathrm{C}$, its yield strength and tensile strength will be higher than the yield strength and tensile strength of $10^{\circ} \mathrm{C}$ at the test. Besides, safety factor redundancy is usually used in the design of mechanical products. From the above two point of view, the screw connection should be overloaded when it breaks. Overload is a necessary condition for fracture of screw connection.

In addition, when the material is brittle under uniaxial tension or compressive stress, the cross section should be in the 45 degree angle to the axial tension and compression stress. However, the fracture of the screw joint is relatively flat, and the angle is 90 degrees into the axial direction. From Fig 2, it is known that the fracture source is at the edge of the screw joint rather than the center of the screw. From this we can infer that the screw is subjected to greater bending stress during the operation of the hoist, which results in overload. The material breaks from the maximum tensile stress at the outside of the rod under the bending stress state, and then occurs at a very rapid rate of cleavage propagation fracture, forming a radial crack propagation texture.

In a word, the low temperature brittle fracture of the screw is mainly caused by too large unbalanced load due to improper operation. The incorrect heat treatment process of the screw is one of the reasons for its fracture. It is suggested to strengthen the operation management of crane construction and strictly prohibit illegal operation.

\section{Conclusions}

(1) By analyzing the fracture surface, it is found that the fracture of the connecting rod is brittle cleavage.

(2) The microstructure of the specimen is coarse Widmanstatten . which results in low number of its mechanical properties. The screw connecting rod are not one of the important factors to produce brittle fracture at low temperature without proper heat treatment.

(3) The low temperature brittle fracture of the screw is mainly caused by too large unbalanced load due to improper operation. The incorrect heat treatment process of the screw is one of the reasons for its fracture.

\section{References}

[1] F. Erdogan, G. C. Sih. Journal of Basic Engineering, 1963, 85(4):527.

[2] Fischer K F. Naturwissenschaften, 1979, 41(3):72-72.

[3] Shuai G, Ren X C, Gang C, et al. Chinese Journal of Engineering, 2016.

[4] Cherepanov G P, Peabody A L, Dewit R, et al. 1982, 49(4):673-674.

[5] Yang J, Goldstein J I. Meteoritics \& Planetary Science Supplement, 2003, 38.

[6]Todorov R P, Khristov K G. Metal Science \& Heat Treatment, 2004, 46(1-2):49-53.

[7] Leont'Ev B A, Buzovskii Y N. Metal Science \& Heat Treatment, 1968, 10(11):930-932.

[8] Zhao M, Liu J, Yang X. Transactions of Metal Heat Treatment, 1995.

[9] Boumerzoug Z, Derfouf C, Baudin T. Engineering, 2010, 02(7):502-506.

[10]SAC/TC 183.GB/T 699-2015[S].The National Standard of China. Standards Press of China.2016:11. 